\title{
Simulations meet Machine Learning in Structural Biology
}

\author{
Adrià Pérez ${ }^{\mathrm{a}}$, Gerard Martínez-Rosell ${ }^{\mathrm{a}}$, Gianni De Fabritiis ${ }^{\mathrm{a}, \mathrm{b}}$ \\ ${ }^{a}$ Computational Biophysiscs Laboratory (GRIB-IMIM), Universitat Pompeu Fabra, \\ Barcelona Biomedical Research Park (PRBB), Doctor Aiguader 88, 08003 Barcelona, \\ Spain \\ ${ }^{b}$ Institució Catalana de Recerca i Estudis Avanats (ICREA), Passeig Lluis Companys 23, \\ Barcelona 08010, Spain
}

\begin{abstract}
Classical molecular dynamics (MD) simulations will be able to reach sampling in the second timescale within five years, producing petabytes of simulation data at current force field accuracy. Notwithstanding this, MD will still be in the regime of low-throughput, high-latency predictions with average accuracy. We envisage that machine learning (ML) will be able to solve both the accuracy and time-to-prediction problem by learning predictive models using expensive simulation data. The synergies between classical, quantum simulations and ML methods, such as artificial neural networks, have the potential to drastically reshape the way we make predictions in computational structural biology and drug discovery.
\end{abstract}

\section{Highlights}

- Classical MD will reach the second timescale and generate petabytes of data within five years.

- Accuracy and cost will still restrict simulations to approximate results and high-latency predictions.

- Machine learning has the potential to deliver more accurate and faster predictions by learning predictive models from expensive simulations.

Email address: gianni.defabritiis@upf.edu (Gianni De Fabritiis ) 
Signs of this paradigm change are already present today in quantum calculations.

\section{Introduction}

Molecular dynamics (MD) simulations are one of the predominant techniques to study protein dynamics. MD is often used to capture dynamical processes of proteins across different timescales with atomistic details in order to rationalize biological phenomena. Despite the potential to become a surrogate model of real protein dynamics, some important issues still remain to be solved, mainly: i) high computational cost and sampling limitations [1] and ii) force field accuracy [2, 3, 4].

Classical MD simulations constitute a balance between accuracy and efficiency. For example, quantum-level phenomena such as enzymatic reactions, polarizability and proton transfers are neglected in exchange for computational speed. Commonly used force fields, based on a parameterization of a closed form potential, are fast to compute, but use approximations that forfeit accuracy. The extent to which these limitations may affect the validity of the results depends on the system and the biological question at hand. Quantum mechanics (QM) calculations can be used to obtain an accurate description of a molecule, but are computationally demanding and very limited in terms of sampling. Ideally, one would like to simulate at quantum level accuracy, which describes the physics and chemistry precisely, but at the sampling regime of current classical simulations.

The first simulation of protein dynamics dates from 1977 and consisted of a 9.2 ps trajectory of the bovine pancreatic trypsin inhibitor (BPTI) in vacuum [5]. In 2010, [6] reported a 1 millisecond trajectory of the same protein in explicit solvent, which constitutes a 100 million increase in trajectory length compared to the first simulation. In 30 years, MD simulations have increased sampling capabilities over 8 orders of magnitude, with increasing accuracy in the force fields [2, 3, 4]. In the last 10 years, MD has evolved from single simulation [7, 8, 9] to high-throughput molecular dynamics studies [10, 11, 12, 13, 14, 15, 16], where hundreds of microseconds of simulations are computed in independent trajectories to obtain converged statistics. Software and hardware innovations, such as the implementation of MD codes for GPUs [17, 18, 19, 20], distributed computing projects like Folding@home [21], GPUGRID [22] and the development of special-purpose supercomputers like ANTON [23], are steadily decreasing the computational 
cost of molecular simulations. Additionally, the development of adaptive sampling schemes have introduced more efficient ways to sample conformational space, decreasing the amount of simulations needed [24, 25, 26].

In a recent review we estimated that MD would reach seconds of aggregated sampling using commodity hardware by 2022 [27] [Fig 1a], generating petabytes of simulation data. For instance, the file size of one second of simulation data of a 60,000-atom system (e.g. a GPCR system) at $0.1 \mathrm{~ns}$ per frame is 7.2 Petabytes (reduced to a third using compressed trajectory file formats). This amount of data constitutes a valuable source of information, but the knowledge extracted from it is mainly used to rationalize a particular protein system at hand, not to generalize it to other systems. In this review, we envision a paradigm change in the near future where expensive simulations (QM and MD) are not used to predict but to learn models, so that further predictions can be drawn using ML approaches. By doing so, the large computational cost required by simulations becomes justifiable, in particular if the results are more accurate by the use of more expensive simulation methods.

\section{Machine learning applied to structural biology}

ML approaches are not new in simulation analysis. For instance, the common analysis pipeline for MD simulations involves dimensionality reduction [28, 29, 30, 31, 32, 33]) and clustering algorithms.

In the last few years, ML applications have grown exponentially. One of the main factors driving this growth is the broad popularization of a particular type of ML called deep neural networks [34, 35]. An artificial neural network $(\mathrm{NN})$ is a simple mathematical framework organized in layers, each of them performing a matrix multiplication and a non-linear function of the input variables $\boldsymbol{x}$. The output of a single neuron $\phi$ in each layer is given by $\phi=f\left(\boldsymbol{w}^{t} \boldsymbol{x}+b\right)$, where $\boldsymbol{w}$ are learnable weights, $b$ is a bias and $f$ is some nonlinear function. NNs can have several to hundred of nested layers and in such cases is called "deep". Given enough parameters, a NN is capable of interpolating any continuous function [36, 37].

The application of NN models in computational biology is steadily increasing [38]. For instance, the Merck molecular activity challenge demonstrated the potential of deep neural network models in drug discovery [39]. DeepTox [40] is a deep learning-based model for toxicity prediction of compounds, winning the Tox21 toxicology prediction challenge in 2014 by a large 
margin. Variational autoencoders [41, a generative flavor of deep NNs, were recently applied to convert discrete representations of molecules to and from a multidimensional continuous representation [42], allowing for efficient search and optimization through open-ended spaces of chemical compounds. Additionally, autoencoders have also been used for dimensionality reduction in MD [43, 44, 45]. VAMPnets [46] fit a Markov state model from the system specific molecular simulation data. NNs have also been used to reproduce the free-energy surface of molecules [47. Deep convolutional neural networks (CNN) [4] have become increasingly popular due to its performance in machine vision, a property that has been exploited by us and others to apply it on structural biology by treating proteins as 3D images. CNNs have been used for ligand binding site detection [49], ligand pose prediction [50], ligand active/inactive classification [51], ligand binding affinity prediction [52] and protein design [53]. Also, the DeepChem software [54] and the MoleculeNet challenge [55] provide multiple featurization algorithms and access to relevant QSAR prediction datasets.

\section{Case 1: ML models to predict quantum forces using QM simulation data}

One important application case of ML is apparent in quantum mechanics. QM simulations are notoriously computationally expensive and, depending on the level of theory, scales poorly with the number of atoms of the system [59]. It is therefore not surprising that there have been efforts to interpolate the QM many-body potential with $\mathrm{NNs}$ to obtain a predictive model of QM forces.

Many studies on approximating QM with NNs were performed previously to the recent $\mathrm{NN}$ resurgence. In particular, Behler et al. 60, 61, 62] contributed significantly to the field for small molecules and on ways to fit quantum observables, e.g. infrared spectra 63]. The initial effort went to provide usable symmetry functions that could guarantee basic physical principles like translational, rotational 64 and invariance on atom reordering to the learned potentials. Transferability, however, was limited until recently [65].

In [65, 66, 67, 68], NNs are trained from QM simulation data to generate the potential energy surface and forces for small molecules, generalizing to unseen molecules, including some preliminary tests on proteins. In the same way as MD force fields do, forces are true derivatives of the interpolated potential energy surface using the gradients of the NN, and can be used to 


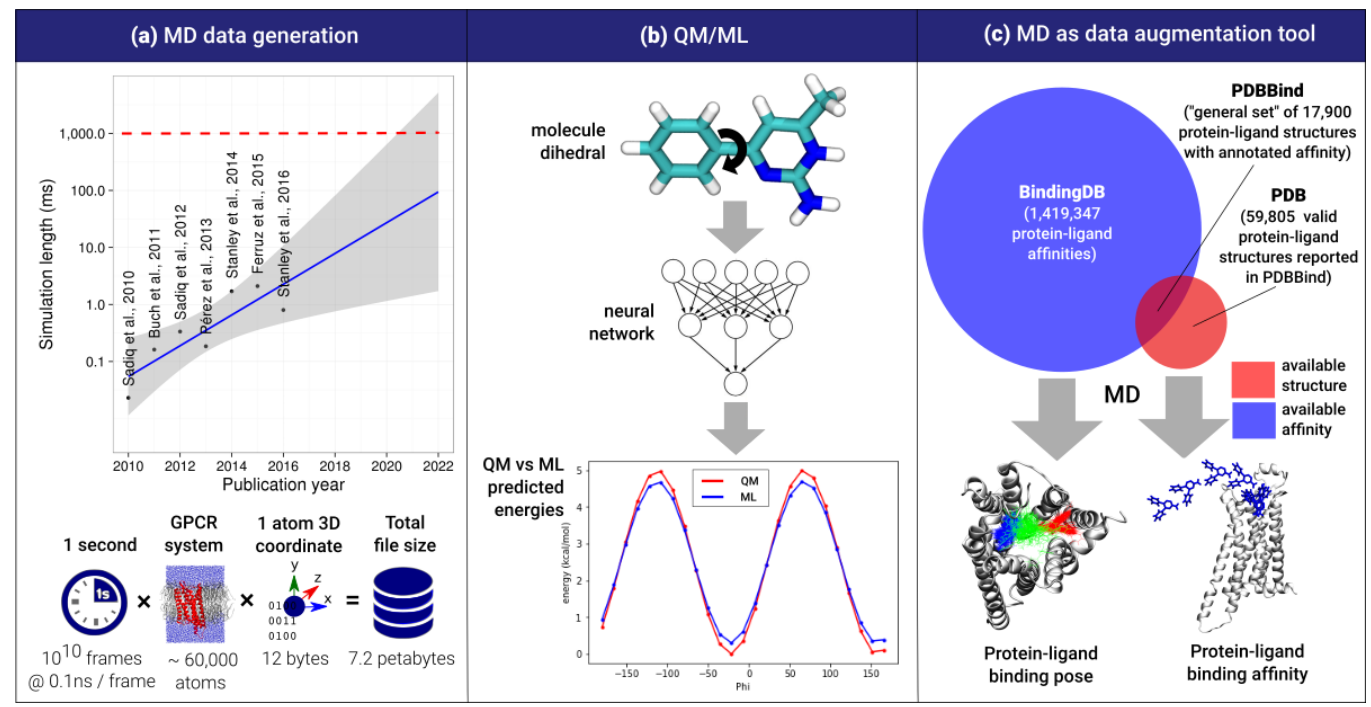

Figure 1: Overview of a combined simulation and machine learning approach. a. MD data generation is expected to reach the second aggregated timescale by 2022 and an output files size of several petabytes by 2022 based on a trend of maximum aggregated time per paper per year using the ACEMD software. Chart adapted from [27]. Referenced publications correspond to [12, 13, 15, 29, 56, 57, 58]. b. A first example of ML replacing QM to predict dihedral energies given a neural network trained with QM simulations. c. An example of data augmentation by MD: augment protein-ligand binding poses for a set of protein-ligand pairs with unknown binding mode; augment binding affinity data for a set of resolved protein-ligand complex structures of unknown affinities.

run dynamics. This guarantees that the forces produced by the NNs yields a conservative field [69]. The QM energy potential is therefore learned with the accuracy of first-principle based methods, using generated datasets for many molecules. The computational cost of generating the datasets is of course very large, but once trained, the $\mathrm{NN}$ inference cost is many orders of magnitude faster than the QM computational model and comparable in costs to standard classical MD [Fig 1b].

\section{Case 2: ML models to predict binding affinities using MD simula- tion data}

MD software for GPU has made simulations of full protein-ligand binding processes possible, allowing the prediction of thermodynamic and kinetic properties [12, 13]. At the moment, a trade-off between accuracy and sampling restricts the applicability of MD compared to other commonly used 
methods used in drug design, like docking, less accurate but significantly faster. Even if the sampling problem is solved via brute force, MD does not currently guarantee that the results are correct because of the approximations of the force fields. The last point can be mitigated by the use of $\mathrm{QM} / \mathrm{ML}$ force fields in the future.

Recently, we explored the use of machine vision NN models for binding affinity prediction. In $K_{\text {DEEP }}$ [52], a ML model is used to predict binding affinities, which consists of a CNN trained on the PDBBind database [70. The method shows an overall good mean correlation (0.82) when tested against the PDBBind's core set. This set contains several targets clustered by sequence similarity, in order to define a representative, non-redundant subset of proteins. For few of these protein clusters, however, the correlation disappears or even becomes negative. This fact might be explained by a lack of training data for specific protein pockets, which ultimately leads to a poor generalization in these cases. $K_{\text {DEEP }}$ is structure-based, i.e. it requires labelled data in the form of the structure of the protein-ligand complex and their affinity. One way to address this issue would be to extend the available training datasets by obtaining new affinity data or structures, either experimentally or computationally (Fig. 11F). Experiments are of course a possibility, viable for pharmaceutical companies and some academic groups. Here we prefer to look at the computational options which can be more automated in active learning methods and are subjected to be exponentially cheaper in the future.

A potential synergy between MD and ML would improve the accuracy of predictive NN models, delivering predictions several orders of magnitude faster than simulations. This level of performance is needed for large prediction studies in drug design, where thousands of molecules need to be evaluated, such as in virtual screening. As for training $K_{\text {DEEP }}$, the two most popular binding affinity databases are PDBBind [70] and Binding MOAD [71. PDBind's latest release (v2017) screened the 124,962 structures in the PDB database [72] (as in Jan 1st, 2017) and identified 59,805 valid molecular complex structures into four main categories: protein-small ligand, nucleic acid-small ligand, protein-nucleic acid and protein-protein complexes. From this set of structures, they defined the general set, providing binding affinity data (KD/KI and IC50) for a total of 17,900 biomolecular complexes in the PDB database, including protein-ligand (14,761), nucleic acid-ligand (121), protein-nucleic acid $(837)$, and protein-protein complexes $(2,181)$. The other dataset, Binding MOAD, contains binding information for 9142 structures, 
being 6862 of them overlapped with PDBBind. This makes a total of 20,065 co-crystal structures with binding data, out of the 59,805 complex structures detected in the PDB. A naive example of synergy could be to increase the available affinity data for the remaining 39,740 structures by simulations. This, however would be very expensive and arguably impractical in a prediction study. Yet in the context of generating a database for training NN models, it only needs to be performed once, and possibly at very high accuracy using QM/ML-based force fields to obtain very accurate data. The resulting NN will be used for predictions. Another possible example comes from the BindingDB dataset [73] which contains about 1,419,347 binding data for 7,000 proteins and over 635,301 drug-like molecules, but for most of the protein-ligand pairs there is no co-crystal structural information. To fill up this gap, simulations could be used to predict ligand binding poses. As a rough estimation, if approximately $10 \mu$ s are needed to obtain the ligand binding pose for a pair of protein-ligand using adaptive sampling methods [26], with 1s of aggregate time one could generate 100,000 new predicted proteinligand structures over the course of one year[27]. This augmented dataset build at high computational and time cost is then used for learning fast predictive models, e.g. $K_{\text {DEEP }}$.

\section{Discussion}

In this article we illustrate how generated data produced by simulations might be used to develop new and better predictive ML models. Generation of datasets is not hampered by fast return times, which means that better simulation methods can be used, while ML is used to obtain fast predictions. One existing example of this approach are QM simulations of biomolecules, used to generate data for learning a NN QM potential, a paradigm that could improve on classical force fields in the near future. A further possible exam-

ple, build upon the experience obtained in $K_{\mathrm{DEEP}}$, is where simulations are used as a data augmentation tool, delegating the binding affinity prediction to ML-based methods.

\section{Acknowledgements}

The authors thank Acellera Ltd. for funding. G.D.F. acknowledges support from MINECO (BIO2017-82628-P) and FEDER, as well as 'Unidad de Excelencia María de Maeztu', funded by MINECO (MDM-2014-0370). The 
authors thank the European Unions Horizon 2020 research and innovation programme under grant agreement No 675451 (CompBioMed project).

\section{References}

\section{References}

[1] P. L. Freddolino, C. B. Harrison, Y. Liu, and K. Schulten, "Challenges in protein-folding simulations," Nat. Phys., vol. 6, no. 10, pp. 751-758, 2010 .

[2] K. A. Beauchamp, Y. S. Lin, R. Das, and V. S. Pande, "Are protein force fields getting better? A systematic benchmark on 524 diverse NMR measurements," J. Chem. Theory Comput., vol. 8, no. 4, pp. 1409-1414, 2012 .

[3] K. Lindorff-Larsen, P. Maragakis, S. Piana, M. P. Eastwood, R. O. Dror, and D. E. Shaw, "Systematic validation of protein force fields against experimental data," PLoS ONE, vol. 7, no. 2, 2012.

[4] S. Piana, J. L. Klepeis, and D. E. Shaw, "Assessing the accuracy of physical models used in protein-folding simulations: Quantitative evidence from long molecular dynamics simulations," Curr. Opin. Struct. Biol., vol. 24, no. 1, pp. 98-105, 2014.

[5] J. A. McCammon, B. R. Gelin, and M. Karplus, "Dynamics of folded proteins," Nature, vol. 267, no. 5612, pp. 585-590, 1977.

[6] D. E. Shaw, P. Maragakis, K. Lindorff-Larsen, S. Piana, R. O. Dror, M. P. Eastwood, J. A. Bank, J. M. Jumper, J. K. Salmon, Y. Shan, and W. Wriggers, "Atomic-level characterization of the structural dynamics of proteins," Science, vol. 330, no. 6002, pp. 341-346, 2010.

[7] Y. Duan, "Pathways to a Protein Folding Intermediate Observed in a 1-Microsecond Simulation in Aqueous Solution," Science, vol. 282, no. 5389, pp. 740-744, 1998.

[8] A. Grossfield, M. C. Pitman, S. E. Feller, O. Soubias, and K. Gawrisch, "Internal Hydration Increases during Activation of the G-ProteinCoupled Receptor Rhodopsin," J. Mol. Biol., vol. 381, no. 2, pp. 478486, 2008. 
[9] R. O. Dror, D. H. Arlow, D. W. Borhani, M. O. Jensen, S. Piana, and D. E. Shaw, "Identification of two distinct inactive conformations of the 2-adrenergic receptor reconciles structural and biochemical observations," Proc. Natl. Acad. Sci., vol. 106, no. 12, pp. 4689-4694, 2009.

[10] C. D. Snow, B. Zagrovic, and V. S. Pande, "The Trp cage: Folding kinetics and unfolded state topology via molecular dynamics simulations," J. Am. Chem. Soc., vol. 124, no. 49, pp. 14548-14549, 2002.

[11] F. Noe, C. Schutte, E. Vanden-Eijnden, L. Reich, and T. R. Weikl, "Constructing the equilibrium ensemble of folding pathways from short off-equilibrium simulations," Proc. Natl. Acad. Sci., vol. 106, no. 45, pp. 19011-19016, 2009.

[12] I. Buch, T. Giorgino, and G. De Fabritiis, "Complete reconstruction of an enzyme-inhibitor binding process by molecular dynamics simulations," Proc. Natl. Acad. Sci., vol. 108, no. 25, pp. 10184-10189, 2011.

[13] N. Ferruz, M. J. Harvey, J. Mestres, and G. De Fabritiis, "Insights from Fragment Hit Binding Assays by Molecular Simulations," J. Chem. Inf. Model., vol. 55, no. 10, pp. 2200-2205, 2015.

[14] A. C. Pan, H. Xu, T. Palpant, and D. E. Shaw, "Quantitative Characterization of the Binding and Unbinding of Millimolar Drug Fragments with Molecular Dynamics Simulations," J. Chem. Theory Comput., vol. 13, no. 7, pp. 3372-3377, 2017.

[15] N. Stanley, L. Pardo, and G. D. Fabritiis, "The pathway of ligand entry from the membrane bilayer to a lipid G protein-coupled receptor," Sci. Rep., vol. 6, no. 1, p. 22639, 2016.

[16] N. Plattner, S. Doerr, G. De Fabritiis, and F. Noé, "Complete proteinprotein association kinetics in atomic detail revealed by molecular dynamics simulations and Markov modelling," Nat. Chem., 2017.

- Plattner et al. managed to simulate Barnse-Barstar protein-protein association.

[17] M. S. Friedrichs, P. Eastman, V. Vaidyanathan, M. Houston, S. Legrand, A. L. Beberg, D. L. Ensign, C. M. Bruns, and V. S. Pande, "Accelerating molecular dynamic simulation on graphics processing units," J. Comput. Chem., vol. 30, no. 6, pp. 864-872, 2009. 
[18] M. J. Harvey and G. De Fabritiis, "An implementation of the smooth particle mesh Ewald method on GPU hardware," J. Chem. Theory Comput., vol. 5, no. 9, pp. 2371-2377, 2009.

[19] M. J. Harvey, G. Giupponi, and G. De Fabritiis, "ACEMD: Accelerating biomolecular dynamics in the microsecond time scale," J. Chem. Theory Comput., vol. 5, no. 6, pp. 1632-1639, 2009.

[20] P. Eastman, J. Swails, J. D. Chodera, R. T. McGibbon, Y. Zhao, K. A. Beauchamp, L. P. Wang, A. C. Simmonett, M. P. Harrigan, C. D. Stern, et al., "OpenMM 7: Rapid development of high performance algorithms for molecular dynamics," PLoS Comput. Biol., vol. 13, no. 7, 2017.

[21] M. Shirts and V. S. Pande, "COMPUTING: Screen Savers of the World Unite!," Science, vol. 290, no. 5498, pp. 1903-4, 2000.

[22] I. Buch, M. J. Harvey, T. Giorgino, D. P. Anderson, and G. De Fabritiis, "High-throughput all-atom molecular dynamics simulations using distributed computing," J. Chem. Inf. Model., vol. 50, no. 3, pp. 397-403, 2010.

[23] D. E. Shaw, J. C. Chao, M. P. Eastwood, J. Gagliardo, J. P. Grossman, C. R. Ho, D. J. Lerardi, I. Kolossváry, J. L. Klepeis, T. Layman, et al., "Anton, a special-purpose machine for molecular dynamics simulation," Commun. $A C M$, vol. 51, no. 7, p. 91, 2008.

[24] N. Singhal and V. S. Pande, "Error analysis and efficient sampling in Markovian state models for molecular dynamics," J. Chem. Phys., vol. 123, no. 20, 2005 .

[25] N. S. Hinrichs and V. S. Pande, "Calculation of the distribution of eigenvalues and eigenvectors in Markovian state models for molecular dynamics," $J$. Chem. Phys., vol. 126, no. 24, 2007.

[26] S. Doerr and G. De Fabritiis, "On-the-fly learning and sampling of ligand binding by high-throughput molecular simulations," J. Chem. Theory Comput., vol. 10, no. 5, pp. 2064-2069, 2014.

[27] G. Martínez-Rosell, T. Giorgino, M. J. Harvey, and G. de Fabritiis, "Drug Discovery and Molecular Dynamics: Methods, Applications and Perspective Beyond the Second Timescale," Curr. Top. Med. Chem., vol. 17, no. 23, pp. 2617-2625, 2017. 
[28] F. Noé and F. Nüske, "A Variational Approach to Modeling Slow Processes in Stochastic Dynamical Systems," Multiscale Model. Simul., vol. 11, no. 2, pp. 635-655, 2013.

[29] G. Pérez-Hernández, F. Paul, T. Giorgino, G. De Fabritiis, and F. Noé, "Identification of slow molecular order parameters for Markov model construction," J. Chem. Phys., vol. 139, no. 1, 2013.

[30] C. R. Schwantes and V. S. Pande, "Improvements in Markov State Model construction reveal many non-native interactions in the folding of NTL9," $J$. Chem. Theory Comput., vol. 9, no. 4, pp. 2000-2009, 2013.

[31] A. Amadei, A. B. M. Linssen, and H. J. C. Berendsen, "Essential dynamics of proteins," Proteins Struct. Funct. Bioinforma., vol. 17, no. 4, pp. 412-425, 1993.

[32] O. F. Lange and H. Grubmüller, "Can principal components yield a dimension reduced description of protein dynamics on long time scales?," J. Phys. Chem. $B$, vol. 110, no. 45, pp. 22842-22852, 2006.

[33] C. C. David and D. J. Jacobs, "Principal component analysis: A method for determining the essential dynamics of proteins," Methods Mol. Biol., vol. 1084, pp. 193-226, 2014.

[34] Y. LeCun, Y. Bengio, and G. Hinton, "Deep learning.," Nature, vol. 521, no. 7553 , pp. $436-44,2015$.

[35] J. Schmidhuber, "Deep Learning in neural networks: An overview," Neural Networks, vol. 61, pp. 85-117, 2015.

[36] K. Hornik, "Approximation capabilities of multilayer feedforward networks," Neural Networks, vol. 4, no. 2, pp. 251-257, 1991.

[37] A. Andoni, R. Panigrahy, G. Valiant, and L. Zhang, "Learning Polynomials with Neural Networks," Proceedings of the 31 st International Conference on Machine Learning, vol. 330, pp. 1-9, 2014.

[38] C. Angermueller, T. Pärnamaa, L. Parts, and O. Stegle, "Deep learning for computational biology," Mol. Syst. Biol., vol. 12, no. 7, p. 878, 2016.

[39] G. E. Dahl, N. Jaitly, and R. Salakhutdinov, "Multi-task Neural Networks for QSAR Predictions," arXiv, pp. 1-21, 2014. 
[40] A. Mayr, G. Klambauer, T. Unterthiner, and S. Hochreiter, "DeepTox: Toxicity Prediction using Deep Learning," Front. Environ. Sci., vol. 3, 2016.

[41] D. P. Kingma and M. Welling, "Auto-Encoding Variational Bayes," arXiv, pp. 1-14, 2013.

[42] R. Gómez-Bombarelli, D. Duvenaud, J. M. Hernández-Lobato, J. AguileraIparraguirre, T. D. Hirzel, R. P. Adams, and A. Aspuru-Guzik, "Automatic chemical design using a data-driven continuous representation of molecules," arXiv, pp. 1-28, 2016.

[43] C. Wehmeyer and F. Noe, "Time-lagged autoencoders: Deep learning of slow collective variables for molecular kinetics," arXiv, pp. 1-8, 2017.

[44] S. Doerr, I. Ariz-Extreme, M. J. Harvey, and G. De Fabritiis, "Dimensionality reduction methods for molecular simulations," arXiv, pp. 1-11, 2017.

[45] C. X. Hernndez, H. K. Wayment-Steele, M. M. Sultan, B. E. Husic, and V. S. Pande, "Variational Encoding of Complex Dynamics," arXiv, pp. 1-12, 2017.

[46] A. Mardt, L. Pasquali, H. Wu, and F. Noe, "VAMPnets: Deep learning of molecular kinetics," arXiv, pp. 1-14, 2017.

[47] E. Schneider, L. Dai, R. Q. Topper, C. Drechsel-Grau, and M. E. Tuckerman, "Stochastic Neural Network Approach for Learning High-Dimensional Free Energy Surfaces," Phys. Rev. Lett., vol. 119, no. 15, 2017.

[48] A. Krizhevsky, I. Sulskever, and G. E. Hinton, "ImageNet Classification with Deep Convolutional Neural Networks," Adv. Neural Inf. Process. Syst., vol. 60, no. 6, pp. 84-90, 2012.

[49] J. Jiménez, S. Doerr, G. Martínez-Rosell, A. S. Rose, and G. De Fabritiis, "DeepSite: protein-binding site predictor using 3D-convolutional neural networks," Bioinformatics, vol. 33, no. 19, pp. 3036-3042, 2017.

- DeepSite is a deep convolutional neural network trained with protein structural data, treating proteins as $3 \mathrm{D}$ images. The network predicts the presence of druggable pockets, and demonstrates superior performance than the stateof-the-art.

[50] M. Ragoza, J. Hochuli, E. Idrobo, J. Sunseri, and D. R. Koes, "ProteinLigand Scoring with Convolutional Neural Networks," J. Chem. Inf. Model., vol. 57, no. 4, pp. 942-957, 2017. 
[51] I. Wallach, M. Dzamba, and A. Heifets, "AtomNet: A Deep Convolutional Neural Network for Bioactivity Prediction in Structure-based Drug Discovery," arXiv, pp. 1-11, 2015.

[52] J. Jiménez, M. Škalič, G. Martínez-Rosell, and G. De Fabritiis, " $K_{\text {DEEP: }}$ Protein-ligand absolute binding affinity prediction via 3D-convolutional neural networks," J. Chem. Inf. Model, pp. 1-26, 2018. Just Accepted Manuscript

- $K_{\text {DEEP }}$ is a deep convolutional neural network trained over the PDBBind's dataset, treating proteins as 3D images, to perform predictions on proteinligand binding affinity.

[53] W. Torng and R. B. Altman, "3D deep convolutional neural networks for amino acid environment similarity analysis," BMC Bioinformatics, vol. 18, no. $1,2017$.

[54] DeepChem, "Deepchem, a python library democratizing deep learning for science." URL: http://www.deepchem.io Accessed: 2017-09-21

- DeepChem is a Python library that aims to provide a high-quality open source tool for deep learning applied on to computational chemistry, making more accesible the usage of deep neural networks for drug discovery, materials science, quantum chemistry and biology.

[55] Z. Wu, B. Ramsundar, E. N. Feinberg, J. Gomes, C. Geniesse, A. S. Pappu, K. Leswing, and V. Pande, "MoleculeNet: A Benchmark for Molecular Machine Learning," arXiv, pp. 1-39, 2017.

[56] S. K. Sadiq and G. de Fabritiis, "Explicit solvent dynamics and energetics of HIV-1 protease flap opening and closing," Proteins Struct. Funct. Bioinforma., vol. 78, no. 14, pp. 2873-2885, 2010.

[57] S. K. Sadiq, F. Noe, and G. De Fabritiis, "Kinetic characterization of the critical step in HIV-1 protease maturation," Proc. Natl. Acad. Sci., vol. 109, no. 50, pp. 20449-20454, 2012.

[58] N. Stanley, S. Esteban-Martín, and G. De Fabritiis, "Kinetic modulation of a disordered protein domain by phosphorylation," Nat. Commun., vol. 5, 2014.

[59] G. Carleo and M. Troyer, "Solving the quantum many-body problem with artificial neural networks," Science, vol. 355, no. 6325, pp. 602-606, 2017.

[60] J. Behler and M. Parrinello, "Generalized neural-network representation of high-dimensional potential-energy surfaces," Phys. Rev. Lett., vol. 98, no. 14, 
2007.

-• One of the first contribution on learning the potential energy surface of molecules using neural networks.

[61] J. Behler, "Atom-centered symmetry functions for constructing highdimensional neural network potentials," J. Chem. Phys., vol. 134, no. 7, p. 074106, 2011.

[62] J. Behler, "Constructing high-dimensional neural network potentials: A tutorial review," Int. J. Quantum Chem., vol. 115, pp. 1032-1050, 2015.

[63] M. Gastegger, J. Behler, and P. Marquetand, "Machine Learning Molecular Dynamics for the Simulation of Infrared Spectra," Chem. Sci., vol. 8, pp. 6924-6935, 2017.

[64] W. Boomsma and J. Frellsen, "Spherical convolutions and their application in molecular modelling," Neural Information Processing Systems (NIPS), 2017.

[65] J. S. Smith, O. Isayev, and A. E. Roitberg, "ANI-1: an extensible neural network potential with DFT accuracy at force field computational cost," Chem. Sci., vol. 8, no. 4, pp. 3192-3203, 2017.

•• In this paper they present ANI-1, a neural network trained with QM simulation data to generate the potential energy surface and forces for small molecules.

[66] K. Yao, J. E. Herr, D. W. Toth, R. Mcintyre, and J. Parkhill, "The TensorMol-0.1 Model Chemistry: a Neural Network Augmented with LongRange Physics," arXiv, pp. 1-8, 2017.

- TensorMol is a neural network potential trained over quantum mechanics simulations that is able to generate the potential energy surface and forces of small molecules.

[67] L. Zhang, J. Han, H. Wang, R. Car, and W. E, "Deep Potential Molecular Dynamics: a scalable model with the accuracy of quantum mechanics," arXiv, pp. 1-22, 2017.

[68] F. A. Faber, L. Hutchison, B. Huang, J. Gilmer, S. S. Schoenholz, G. E. Dahl, O. Vinyals, S. Kearnes, P. F. Riley, and O. A. Von Lilienfeld, "Prediction Errors of Molecular Machine Learning Models Lower than Hybrid DFT Error," J. Chem. Theory Comput., vol. 13, no. 11, pp. 5255-5264, 2017. 
[69] S. Chmiela, A. Tkatchenko, H. E. Sauceda, I. Poltavsky, K. T. Schütt, and K.R. Müller, "Machine learning of accurate energy-conserving molecular force fields," Sci. Adv., vol. 3, no. 5, 2017.

[70] Z. Liu, M. Su, L. Han, J. Liu, Q. Yang, Y. Li, and R. Wang, "Forging the Basis for Developing Protein-Ligand Interaction Scoring Functions," Acc. Chem. Res., vol. 50, no. 2, pp. 302-309, 2017.

[71] A. Ahmed, R. D. Smith, J. J. Clark, J. B. Jr, and H. A. Carlson, "Recent improvements to Binding MOAD: A resource for protein-ligand Binding affinities and structures," Nucleic Acids Res., vol. 43, no. D1, pp. D465-D469, 2015.

[72] H. M. Berman, J. Westbrook, Z. Feng, G. Gilliland, T. N. Bhat, H. Weissig, I. N. Shindyalov, and P. E. Bourne, "The Protein Data Bank," Nucleic Acids Res., vol. 28, no. 1, pp. 235-242, 2000.

[73] M. K. Gilson, T. Liu, M. Baitaluk, G. Nicola, L. Hwang, and J. Chong, "BindingDB in 2015: A public database for medicinal chemistry, computational chemistry and systems pharmacology," Nucleic Acids Res., vol. 44, no. D1, pp. D1045-D1053, 2016. 